\title{
Clinicopathological Analysis of Undifferentiated Malignant Neoplasms of The Sinonasal Tract
}

\author{
Ashok kumar $\mathrm{S}^{1}$, Srigayathri $\mathrm{S}^{2}$ and Geetha Devadas ${ }^{1}$ \\ ${ }^{1}$ Department of pathology, Govt Stanley Medical college, Chennai, TN. India \\ ${ }^{2}$ Sri Ramachandra Medical College And Research Institute, Chennai, TN, India
}

\section{ABSTRACT}

Background: Sinonasal tumours are characterized by low incidence, non specific symptoms, and late presentation. Aggressive, nonsquamous cell epithelial and nonepithelial malignant neoplasms of varying histogenesis occurring in sinonasal region are grouped under the term undifferentiated malignant neoplasms. Frequently, these undifferentiated malignancies share clinical and light microscopic features, which make differentiation of one from the other virtually impossible without the use of adjunct immunohistochemical analysis .

Methods: Undifferentiated malignant sinonasal tumors biopsied or surgically excised over a period of 5 years were studied.

Result: We encountered 22 cases of undifferentiated malignant sinonasal tumors with an incidence of $0.05 \%$, with a mean age of 41 to 50 years and the male to female ratio was 2.1:1. Epithelial tumors (18 cases) constituting $81.82 \%$ predominated over non epithelial tumors (4 cases) constituting $18.18 \%$ with a ratio of $4.5: 1.16$ tumors involved the nasal cavity $(72.7 \%)$ and 6 involved paranasal sinuses $(27.3 \%)$. The most common clinical presentation was mass in the nose 19 cases $(86.36 \%)$. The most common Undifferentiated malignant tumor encountered was Sinonasal undifferentiated carcinoma-10 cases $(45.45 \%)$.

Conclusion: A variety of undifferentiated malignant neoplasms occur in the sinonasal tract with overlapping clinical and pathologic findings. In limited biopsy material, differentiation of these tumor types can be challenging and differentiating these tumors with the help of immunohistoochemistry has clinical importance because advances in therapeutic intervention may increase survival with good quality of life, and in some instances may achieve a cure.

\section{Keywords: Undifferentiated, Malignant, Sinonasal, Neoplasms, Immunohistochemistry}

\section{Introduction}

The nasal cavity and paranasal sinuses including the maxillary, ethmoid, sphenoid and frontal sinuses are collectively referred to as the sinonasal tract. Although the nasal cavity and paranasal sinuses occupy a relatively small anatomic space, they are the site of origin of some of the more complex histologically diverse group of tumors of the entire human body. ${ }^{[1,2]}$

These include neoplasms derived from mucosal epithelium, seromucinous glands, soft tissues, bone, cartilage, neural/ neuroectodermal tissues, hematolymphoid cells and the odontogenic apparatus. Many of the tumours are similar to those found elsewhere in the body but a few such as olfactory neuroblastoma are unique to this site. ${ }^{[3]}$

\section{Materials and Methods}

The surgical specimens received in the Institute of Pathology, Madras Medical College, Chennai from the Upgraded Institute of Otorhinolaryngology, Government General Hospital, Chennai for the period of five years formed the material for this study. Small biopsy specimens and excision biopsy specimens and resection specimens were included. Inadequate or unrepresentative biopsy material was excluded from the study. Informed written consent from the patient was obtained. Ethical committee clearance was obtained.

The clinical features such as age and sex of the patient, site of lesion and type of surgery done were noted.

The tissues were routinely processed and paraffin blocks were made and histological sections of 5 to 6 micrometer were taken in Leica microtome and routinely stained with hematoxylin and eosin stains.

The microscopic analyses were done from all the available slides. These included the histological pattern, cellular features, pleomorphism, mitosis, necrosis, vascularity and secondary changes. Provisional histological differential diagnosis was made. Special stains periodic acid (PAS), reticulin and Masson Fontana were done wherever necessary. For undifferentiated malignant neoplasms, we employed the panel of immunohistochemical markers that include: Cytokeratin, Synaptophysin, Neuronspecific 
Enolase,CD 99,vimentin,CD 45,Desmin,S100,HMB 45. After interpretation final diagnosis was made. Microphotographs were taken.

\section{Result}

We encountered 22 cases of Undifferentiated malignant tumors with an incidence of $0.05 \%$. Undifferentiated malignant tumors occurred in patients with a mean age of 41 to 50 years and the male to female ratio was 2.1:1. Among Undifferentiated malignant tumors, epithelial tumors predominated over non epithelial tumors.

The age range varied from second to ninth decade of life but the peak age of presentation was $5^{\text {th }}$ decade followed by $7^{\text {th }}$ decade as shown in table-1.Males showed a higher incidence than females with a male to female ratio of $2.1: 1$ as shown in table-1.

Out of the 22 cases 16 tumors involved the nasal cavity $(72.7 \%)$ and 6 involved paranasal sinuses $(27.3 \%)$ as shown in table-1.

Amongst paranasal sinuses, maxillary sinus was the commonest site in our study as shown in table-1.The most common tumor encountered was Sinonasal undifferentiated carcinoma(SNUC) 10 cases(45.45\%) as shown in table- 1 . The most common clinical presentation in our study was mass in the nose which constituted 19 cases $(86.36 \%)$ as shown in table-1.

In our study we had 15 cases of undifferentiated carcinoma for which we employed a panel of immunohistochemical markers that included cytokeratin, NSE, synaptophysin, vimentin, desmin, S100, CD99 and CD45. 10 cases showed diffuse, moderate staining for cytokeratin and no reactivity for other markers except for 2 cases which in addition to cytokeratin showed focal reactivity for NSE. All those 10 cases were finally diagnosed as Sinonasal undifferentiated carcinoma (SNUC).

5 cases showed diffuse moderate staining for cytokeratin, NSE, synaptophysin, and were finally categorized as Sinonasal neuroendocrine carcinoma (SNEC).

SNUC was the predominant variant (66.67\%) followed by SNEC (33.33\%) as shown in table-1. We had 5 cases of small round cell tumor (SRCT) for which immunohistochemistry panel was employed.

Among them we observed that 2 cases showed reactivity for NSE, synaptophysin, S100 and was finally diagnosed as esthesioneuroblastoma(ENB) constituting $40 \%$.Among the other 3 cases ,2 cases were diagnosed as PNET which showed reactivity for CD 99 and vimentin. 1 case was diagnosed as SNEC and showed reactivity for cytokeratin, NSE and synaptophysin as shown in table-2.

We had 2 cases of malignant melanoma with a incidence of $1.87 \%$. Both cases presented in the nasal cavities of males aged 40 and 54 years respectively. Histopathology showed features similar to other undifferentiated neoplasms of sinonasal tract showing malignant epithelioid and spindle cells with pleomorphic nuclei and eosinophilic nucleoli. Immunostaining revealed tumor cells which were positive for markers HMB45, S100 confirming the provisional diagnosis of melanoma.

TABLE-1: Distribution of Undifferentiated Malignant Tumors of Nasal Cavity, Paranasal Sinuses According to The Incidence, Sex Ratio, Age and Site of Presentation

\begin{tabular}{|c|c|c|c|c|c|c|c|c|}
\hline \multirow[b]{2}{*}{ Diagnosis } & \multirow[b]{2}{*}{ No.of.cases } & \multirow[b]{2}{*}{$\%$} & \multirow[b]{2}{*}{$M: F$} & \multirow[b]{2}{*}{ Peak age(decade) } & \multicolumn{2}{|c|}{ Nasal cavity } & \multicolumn{2}{|c|}{ PNS } \\
\hline & & & & & No & $\%$ & No & $\%$ \\
\hline \multicolumn{9}{|c|}{ Undifferentiated malignant tumors 22} \\
\hline Melanoma & 2 & 1.87 & M only & $4 \& 6$ & 2 & 3.44 & 0 & 0 \\
\hline Olfactory Neuroblastoma & 2 & 1.87 & $1: 1$ & $2 \& 5$ & 1 & 1.72 & 1 & 2.04 \\
\hline SNUC & 10 & 9.35 & $4: 1$ & 5 & 7 & 12.06 & 3 & 6.12 \\
\hline SNEC & 6 & 5.61 & $1.5: 1$ & $3 \& 5$ & 5 & 8.62 & 1 & 2.04 \\
\hline PNET & 2 & 1.87 & M only & 2 & 1 & 1.72 & 1 & 2.04 \\
\hline
\end{tabular}

TABLE 2: Small Round Cell Tumors (SRCT)

\begin{tabular}{|c|c|}
\hline SRCT & No \\
\hline Esthesioneuroblastoma & 2 \\
\hline PNET & 2 \\
\hline SNEC & 1 \\
\hline Total & $\mathbf{5}$ \\
\hline
\end{tabular}




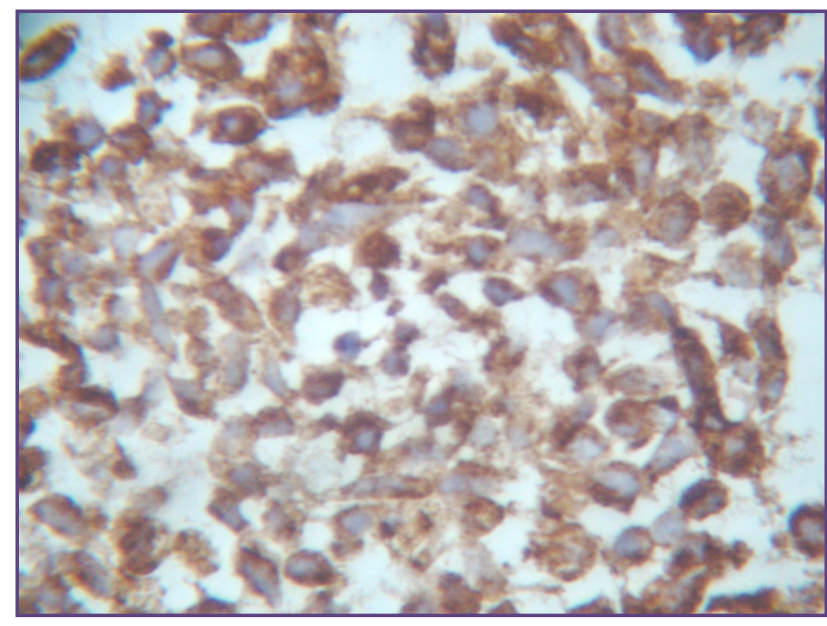

Fig. 1: Neoplastic cells showing positivity for HМB 45 in Malignant melonoma (IHC400x)

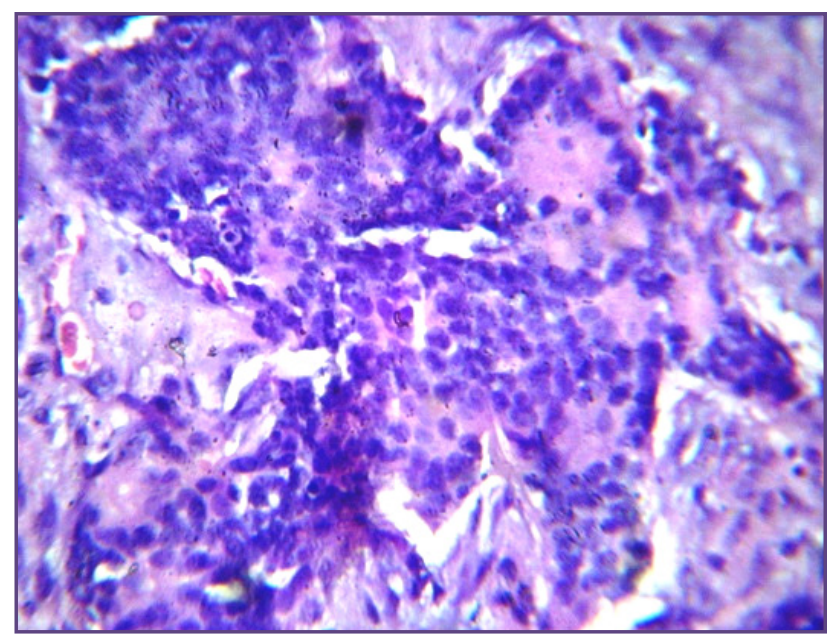

Fig. 3: Neoplasm composed of small round cells with Homer-Wright rosettes in Olf. neuroblastoma (H\&E400X).

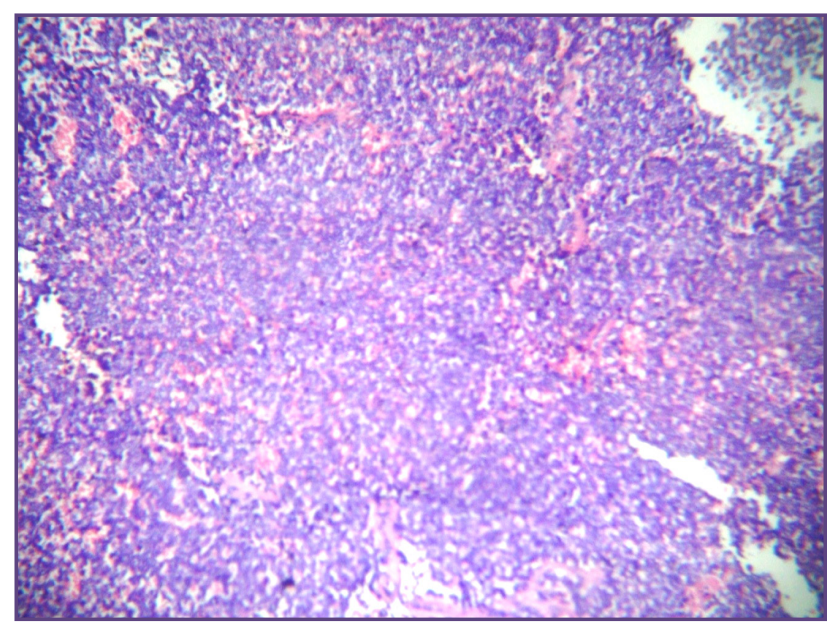

Fig. 5: Neoplasm arranged in diffuse dense cellular sheets in PNET (H\&E100x).

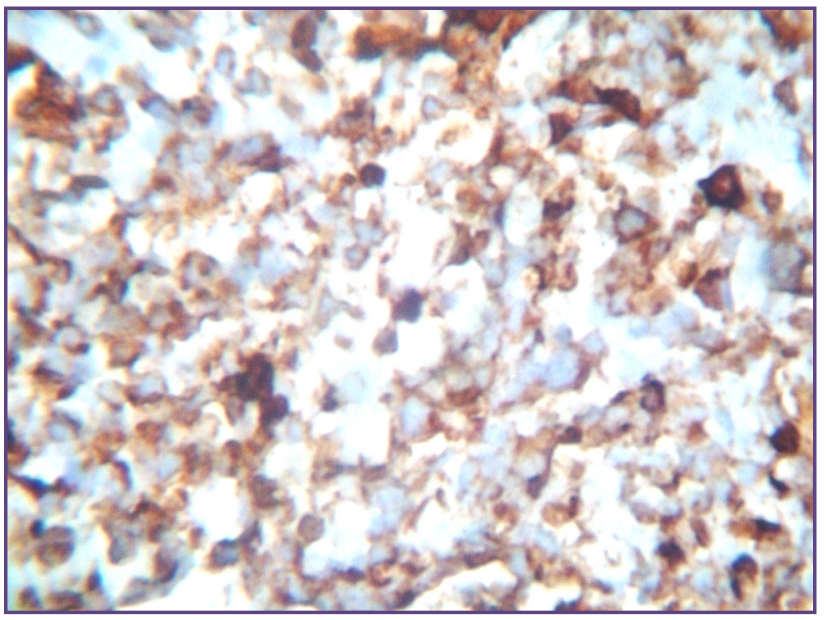

Fig. 2: Neoplastic cells showing membranous positivity for CD99 in case of PNET (IHC400X).

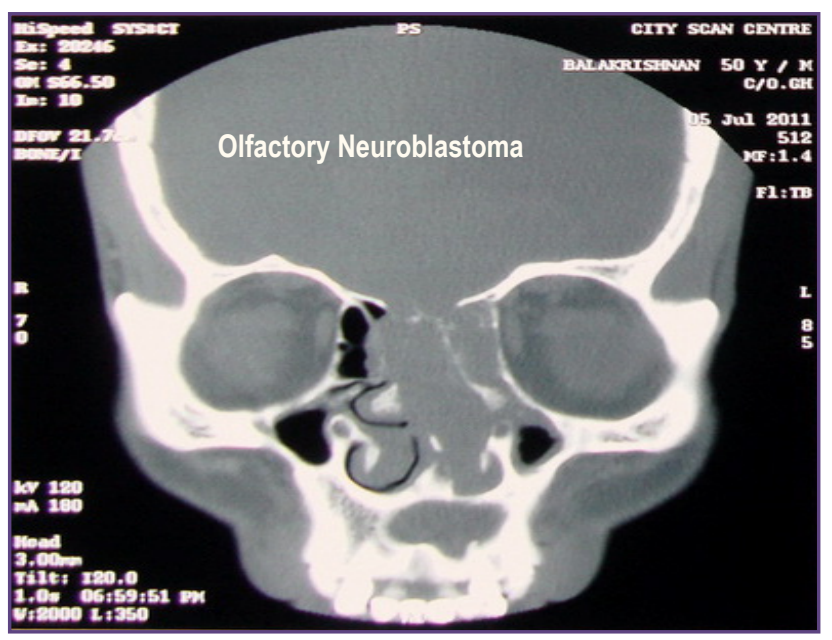

Fig. 4: CT scan showing tumor in the left nasal cavity and ethmoid sinus with Intracranial extension.

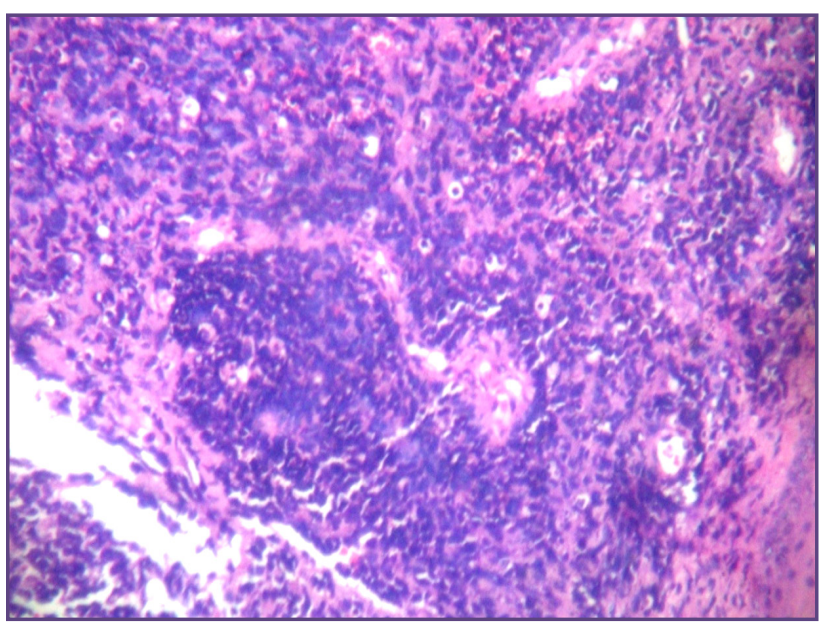

Fig. 6: Cellular neoplasm in solid sheets with focal rosette formation lacking neurofibrillary background (H\&E 100X) SNEC

Annals of Pathology and Laboratory Medicine, Vol. 4, Issue 4, July-August, 2017 


\section{Discussion}

The present clinicopathological study of sinonasal tumors includes 22 undifferentiated tumors of nasal cavity and paranasal sinuses for five years. During the period of five years 44730 specimens were received at the general surgical pathology laboratory of our institute. Out of which 22 were undifferentiated sinonasal tumors with incidence of undifferentiated sinonasal tumors representing $0.05 \%$. The rare nature of the tumors is almost a universal finding. ${ }^{[4,5,6,7]}$

Epithelial tumors (18cases) predominated over nonepithelial tumors (4 cases) constituted $81.82 \%$. The ratio of epithelial to nonepithelial tumors was 4.5:1. Male to female ratio was 2.1:1 similar to other studies (Lilly-Tariah da, 1999; Mundy et al., 1985 ;). Age range was 10 to 70 years with an average of 41 to 50 years. The maximum number of cases were present in nasal cavity $(72.7 \%)$ followed by paranasal sinuses $(27.3 \%)$.Among the paranasal sinuses maxillary sinus $(22.72 \%)$ was the commonest site of presentation. Mass in the nose $(86.36 \%)$ was the most common clinical presentation in our study. Both sides (Right\&Left) involved with equal frequency $(50 \%)$.

We encountered 10 cases of sinonasal undifferentiated carcinomas which showed an incidence of $9.35 \%$.Studies by other authors revealed the incidence of sinonasal undifferentiated carcinoma had a varied range of incidence from 1.7 to $17 \%$. We noted a peak incidence in the 5 th decade in our study. Males dominated over females in our analysis.

In a study done in Taiwan by Jeng YM and Sung MT, 36 cases of sinonasal undifferentiated carcinoma was reviewed .They found that median age of presentation was 53 years with a male female ratio of $2: 1$. The most common locations were nasal cavity and ethmoidal sinus. The commonest site in our study was nasal cavity. Histopathology showed either small or large tumor cells and was predominantly arranged in nests, ribbons, thick trabeculae or sheet like pattern and had coarse chromatin, prominent nucleoli and necrosis. ${ }^{[8,9,10]}$

We had 2 cases of malignant melanomawith a incidence of 1.87. Both cases presented in the nasal cavities of males aged 40 and 54 years respectively similar to Zafer et al(2008). Histopathology showed features similar to other undifferentiated neoplasms of sinonasal tract showing malignant epithelioid and spindle cells with pleomorphic nuclei and eosinophilic nucleoli with evidence of melanin deposition.Masson Fontana stain showed positivity in the cytoplasm of tumor cells. Immunostaining revealed tumor cells which were positive for markers HMB45, S100 confirming the diagnosis of melanoma (Fig. 1). ${ }^{[11,12,13]}$

We had 5cases of small round cell tumors (SRCT) and 15 cases of undifferentiated carcinomas in our study. For confirmation and further subcategorization a panel of immunohistochemical markers that include cytokeratin,NSE,synaptophysin, CD99,vimentin,CD45, desmin,S100 and HMB 45 were employed.Among the 5 cases of SRCT, 2 cases showed positivity for NSE, synaptophysin, and S100 confirming the diagnosis of olfactory neuroblastoma. 2 cases showed positivity for CD99 and vimentin and were negative for other markers thus confiming with PNET (Fig.2). ${ }^{[14]}$ One case showed positivity for cytokeratin, NSE, synaptophysin and a final diagnosis of SNEC was made.

The incidence of olfactory neuroblastoma in our study was $1.87 \%$.Of our 2 cases of olfactory neuroblastoma,one presented in a 20 year old female involving the ethmoid sinus and the other in 48 year old male involving the nasal cavity. We found a bimodal peak in age similar to other studies. ${ }^{[15,16]}$ The tumor was disposed in lobules and nests and composed of small round cells with high N/C ratio, small uniform hyperchromatic nuclei with salt and pepper type of chromatin, Homer-Wright rosettes in a fibrillary background with a Hyams grade I (Fig.3). ${ }^{[15,16,17,18]}$ One of our cases had intracranial extension and cervical lymph node metastasis with a Kadish stage C(Fig.4). FNAC of cervical node revealed small round cells with occasional rosette formation confirming metastasis to cervical node.

Our 2 cases of PNET presented in 15 and 17 year old males respectively with an incidence of $1.87 \%$.One presented in the nasal cavity and the other in the maxilla. Histopathology showed diffuse densely cellular sheets of uniform small to medium sized round cells with scant cytoplasm and round nuclei with delicate chromatin (Fig.5).

One small round cell tumor diagnosed as SNEC presented in the nasal cavity of a 48 year old female.

Of the Undifferentiated neoplasms we noted that 10 cases showed diffuse positivity for cytokeratin alone with focal positivity for NSE only in 2 cases with a final diagnosis of SNUC.

5 cases showed diffuse positivity for both cytokeratin and neuroendocrine markers namely NSE, synaptophysin confirming the diagnosis of SNEC.The incidence of SNEC in our study was $5.61 \%$.There was a slight preponderance in males. Nasal cavity was the commonest site and peak age was found in $5^{\text {th }}$ decade.$^{[19]}$ Histopathology showed sheets of large round cells having moderate amount of cytoplasm, fine chromatin with inconspicuous nucleoli and focal rosette formation without fibrillary background (Fig.6). ${ }^{[19,20,21]}$

\section{Conclusion}

Tumours of the nasal cavity and paranasal sinuses are rare pathologies with extremely varied etiopathology, clinical behaviour, treatment and prognosis. The symptoms of the 
neoplastic processes are essentially similar to inflammatory pathology of the sinonasal tract with resultant delay of diagnosis. The clinical and radiological features of masses of nasal cavity and paranasal sinuses are overlapping and often only a provisional diagnosis is possible. Definite diagnosis requires histopathological examination as most of the lesions are inaccessible for fine needle aspiration or FNAC is not recommended because of fear of haemorrhage.

The key in the diagnosis and treatment of sinonasal tumours remains a high index of suspicion and early diagnosis as late presentation and delay in early diagnosis are major constraints to favourable outcome of treatment. This study highlights the characteristics of the rare sinonasal undifferentiated malignancies encountered and emphasizes the importance of Immunohistochemistry(IHC) in distinguishing esthesioneuroblastoma (ENB) from non ENB which has a bearing on prognosis and therapeutic intervention.

\section{Reference}

1. Wenig BM, Pilch BZ. Tumours of upper respiratory tract. In: Fletcher Christopher DM, editor. Diagnostic Histopathology of Tumours.2nded.Vol.1: London: Harcourt publishersLtd; 2000. 87-138.

2. Taxy JB. Upper respiratory tract. In: Damjanor I, Linder J, editors.Anderson's Pathology.10th ed.Vol .1; Missouri: Mosby Year Book; 1996. 1446-1469.

3. Barnes L, Eveson John W, Peter R, Sidransky D. Pathology and Genetics-Head and Neck Tumours.World health Organisation classification Of Tumours. Lyon:IARC Press:2005.9-82, 284.

4. Chukuezi AB, Nwosu JN. Pattern of Nasal and Paranasal sinus tumours in Owerri ,Nigeria. Research Journal of Medical Sciences. 2010; 4:11-14.

5. Panchal L, Vaideeswar P, Kathpal D, Madiwale CV, Prabhat DP. Sino-nasal epithelial tumours: A pathological study of 69 cases. J Postgrad Med .2005; 51:30-5.

6. De Paula Araujo R, Gomes EF, de Menezes DB. Rare sinonasal tumors: Case series and literature review. Braz J Otorhinolaryngol. 2008; 74:307-14.

7. Khan N, Zafar U, Afroz N, Ahmad SS, Hasan SA. Masses of nasal cavity,paranasal sinuses:A clinicopathologic study. Indian journal of otolaryngology and head and neck surgery 2006; 58:259-63.
8. Casiraghi $\mathrm{O}$, Lefèvre $\mathrm{M}$. Undifferentiated malignant round cell tumors of the sinonasal tract and nasopharynx. Ann Pathol. 2009 Sep; 29:296-312.

9. Wenig BM. Undifferentiated malignant neoplasms of the sinonasal tract. Arch Pathol Lab Med. 2009 May; 133:699-712.

10. Ejaz A, Wenig BM. Review Sinonasal undifferentiated carcinoma: clinical and pathologic features and a discussion on classification, cellular differentiation, and differential diagnosis. Adv Anat Pathol. 2005; 12:134-43.

11. López F, Rodrigo JP, Cardesa A. Update on primary head and neck mucosal melanoma. Head \& neck. 2016; 38:147-155.

12. Clifton N, Harrison L, Bradley P J, Jones N S. Malignant melanoma of nasal cavity and paranasal sinuses: report of 24 patients and literature review. Journal of Laryngology and Otology.2011; 125:479-485.

13. Thompson LDR, Wieneke JA, Miettinen M. Sinonasal Tract and Nasopharyngeal Melanomas.A Clinicopathologic Study of 115 Cases With a ProposedStaging System. The American Journal of Surgical Pathology. 2003;27: 594-611.

14. Kawabata M, Yoshifuku K, Sagara Y, Kurono Y.Ewing's sarcoma/primitive neuroectodermal tumour occurring in the maxillary sinus. Rhinology. 2008; 46:75-8.

15. Shah K, Perez-Ordóñez B.Neuroendocrine Neoplasms of the Sinonasal Tract: Neuroendocrine Carcinomas and Olfactory Neuroblastoma. Head Neck Pathol. 2016; 10:85-94.

16. Bragg, Taryn McFadden, Scianna, Joseph , Kassam, Amin, Emami, Bahman. Clinicopathological review: esthesioneuroblastoma. Neurosurgery. 2009; 64:764-770.

17. Theilgaard SA, Buchwald C, Ingeholm P, Larsen SK, Eriksen J: Esthesioneuroblastoma: a Danish demographic study of 40 patients registered between 1978 and 2000. Acta Otolaryngol. 2003; 123:433-9.

18. DulguerovP,AllalAS, CalcaterraTC.Esthesioneuroblastoma: a metaanalysis and review. Lancet Oncol 2001; 2:683-90.

19. Menon S, Pai P, Sengar M, Aggarwal JP, Kane SV.Sinonasal malignancies with neuroendocrine differentiation: case series and review of literature. Indian J Pathol Microbiol. 2010; 53:28-34.

20. Rischin D, Coleman A. Sinonasal Malignancies of Neuroendocrine Origin. Hematol Oncol Clin N Am. 2008; 22:1297-316.

21. Babin E, Rouleau V, Vedrine PO, Toussaint B, de Raucourt $D$. Small cell neuroendocrine carcinoma of the nasal cavity and paranasal sinuses. J Laryngol Otol. 2006; 120:289-97.

*Corresponding author:

Ashok kumar S, Assistant professor, Department of pathology,Govt Stanley Medical college, Chennai, TN- 600001,India,

Phone: +91 04425281351

Email: ashoknithelan@gmail.com

Financial or other Competing Interests: None.

Date of Submission : 16.03.2017

Date of Acceptance : 29.04.2017

Date of Publication : 31.08.2017 